Correction

\title{
Correction: Zhu, S., et al. A New Digital Lake Bathymetry Model Using the Step-Wise Water Recession Method to Generate 3D Lake Bathymetric Maps Based on DEMs. Water 2019, 11, 1151
}

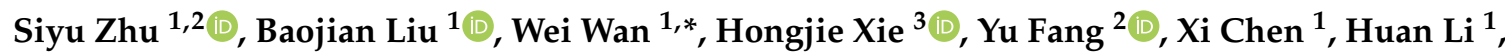 \\ Weizhen Fang ${ }^{1}$, Guoqing Zhang ${ }^{4} \mathbb{D}$, Mingwei Tao ${ }^{2}$ and Yang Hong ${ }^{1,5, *}$ \\ 1 Institute of Remote Sensing and GIS, Peking University, Beijing 100871, China; 13240779409@163.com or \\ zhusy17@mails.tsinghua.edu.cn (S.Z.); liubaojian@pku.edu.cn (B.L.); chenxi928@pku.edu.cn (X.C.); \\ huan.li@pku.edu.cn (H.L.); weizhenfang@pku.edu.cn (W.F.) \\ 2 Hydraulic Engineering, Tsinghua University, Beijing 100083, China; fangyu516@gmail.com (Y.F.); \\ tmw15@mails.tsinghua.edu.cn (M.T.) \\ 3 Department of Geological Sciences, The University of Texas at San Antonio, San Antonio, TX 78249, USA; \\ Hongjie.Xie@utsa.edu \\ 4 Institute of Tibetan Plateau Research, Chinese Academic of Science, Beijing 100101, China; \\ guoqing.zhang@itpcas.ac.cn \\ 5 School of Civil Engineering and Environmental Science, University of Oklahoma, Norman, OK 73019, USA \\ * $\quad$ Correspondence: w.wan@pku.edu.cn (W.W.); yanghong@ou.edu (Y.H.); Tel.: +86-10-6274-4775 (W.W. \& Y.H.)
}

In the published article [1], the authors realized some errors in the affiliation and email address of Yang Hong, and thus wish to make the following revisions:

1. Add the Affiliation 5 "School of Civil Engineering and Environmental Science, University of Oklahoma, Norman, OK 73019, USA" for Yang Hong

2. Change the email address of Yang Hong to yanghong@ou.edu

The authors would like to apologize for any inconvenience caused to the readers by the change. The change does not affect the scientific results. The manuscript will be updated and the original will remain online on the article webpage, with a reference to this Correction.

\section{References}

1. Zhu, S.; Liu, B.; Wan, W.; Xie, H.; Fang, Y.; Chen, X.; Li, H.; Fang, W.; Zhang, G.; Tao, M.; et al. A New Digital Lake Bathymetry Model Using the Step-Wise Water Recession Method to Generate 3D Lake Bathymetric Maps Based on DEMs. Water 2019, 11, 1151. [CrossRef]

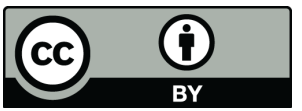

(C) 2019 by the authors. Licensee MDPI, Basel, Switzerland. This article is an open access article distributed under the terms and conditions of the Creative Commons Attribution (CC BY) license (http://creativecommons.org/licenses/by/4.0/). 